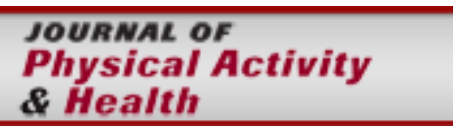

\title{
Insufficient Reporting of Factors Associated with Exercise Referral Scheme Uptake, Attendance and Adherence: A Systematic Review of Reviews.
}

\begin{tabular}{|r|l|}
\hline Journal: & Journal of Physical Activity \& Health \\
\hline Manuscript ID & JPAH.2018-0341.R2 \\
\hline Manuscript Type: & Review \\
\hline Keywords: & physical activity, exercise prescription, program evaluation \\
\hline \multicolumn{2}{|l}{} \\
\end{tabular}

\section{SCHOLARONE \\ Manuscripts}

Accepted author manuscript version reprinted, by permission, from Journal of Physical Activity and Health, 2019, 16 (8), pp. 667-676 https://doi.org/10.1123/jpah.2018-0341 O Human Kinetics, Inc. 
1 Abstract

2 Background: Exercise Referral Schemes (ERS) are prescribed programmes aimed at tackling physical

3 inactivity and associated non-communicable disease. Inconsistencies in reporting, recording and

4 delivering ERS make it challenging to identify what works, why, and for whom.

5 Methods: PRISMA guided this narrative review of reviews. Fifteen electronic databases were

6 searched for systematic reviews of ERS. Reviewers applied inclusion criteria and quality assessed via

7 the AMSTAR tool. Data on uptake, attendance and adherence were extracted.

8 Results: Eleven reviews met the inclusion criteria. AMSTAR quality was medium. Definitions of 9 uptake varied within reviews. Uptake ranged from 35\%-81\%. Groups reported as more likely to take 10 up ERS included, (i) females and (ii) older adults. Attendance was defined variably but ranged from 11 12\%-49\%. Men were more likely to attend ERS. Effect of medical diagnosis upon uptake and 12 attendance was inconsistent. Exercises prescribed were unreported and therefore, adherence to 13 exercise prescriptions was unreported. The influence of theoretically-informed approaches on 14 uptake, attendance and adherence was generally lacking, however, self-determination, peer support 15 and supervision support were reported as influencing attendance.

16 Conclusions: There was insufficient reporting across studies about uptake, attendance and 17 adherence. Complex interventions like ERS require consistent definitions, recording and reporting of 18 these key facets, but this is not evident from the existing literature. 
A Review of Reviews of Exercise Referral Schemes.

\section{Introduction}

The benefits of regular physical activity (PA) are well established $(1,2,3,4)$, notably for adults who engage in at least 150 minutes of moderate intensity activity or 75 minutes of vigorous intensity activity per week, alongside activities that improve muscular strength on at least two days of the week ${ }^{(1)}$. Despite the clear and causal relationship between achieving PA guidelines and health outcomes ${ }^{(1)}$, physical inactivity is rising ${ }^{(5)}$. Inactivity is now the fourth leading risk factor for global mortality (6\% of deaths globally), behind high blood pressure (13\%), tobacco use $(9 \%)$ and high blood glucose $(6 \%)^{(5)}$. Contextually it must be acknowledged that while physical inactivity is a standalone risk factor ${ }^{(5)}$ PA status is a key determinant in moderating the additional risk factors of high blood pressure and high blood glucose levels ${ }^{(5,4)}$.

In recognition of this physical inactivity burden, the UK government has implemented populationwide programmes ${ }^{(6)}$. Additionally, at the individual level, tailored attempts to manage physical inactivity has led to the expansion of exercise referral schemes (ERS). Individual's showing evidence of non-communicable disease coupled with an inactive lifestyle are directly referred by allied health practitioners to independent third party exercise facilities to undertake a structured and prescriptive exercise programme ${ }^{(7)}$. The popularity of the ERS model drove a rapid rise in the number of centres offering referral programmes despite limitations presented within the literature evaluating the implementation of schemes ${ }^{(8)}$ and thereby limited evidence of what works, why and for whom.

Since 1998, a combination of systematic reviews, meta-analyses or narrative reviews of ERS have been published focusing on different constructs surrounding the effectiveness of ERS $(2,9,10,11,12,13,14$, $15,16,17,18,19)$. It is evident from this body of work that ERS is considered an important element in the armoury to increase PA and tackle chronic disease ${ }^{(20)}$. However, the United Kingdom's National Institute for Health and Care Excellence (NICE) have indicated that the varying nature of ERS programmes makes it impossible to differentiate between the effectiveness and acceptability of different schemes ${ }^{(4)}$ in addressing physical inactivity and non-communicable disease. This conclusion 


\section{A Review of Reviews of Exercise Referral Schemes.}

44 is largely due to little evidence of any effectiveness of specific schemes ${ }^{(4)}$. It is crucial therefore, that clear reporting of key ERS components is available to allow for components associated with effectiveness to be replicated in future schemes. Factors such as reporting of referral uptake, attendance and adherence, and the behaviour change techniques (BCT's) underpinning ERS uptake and adherence are key components to understand for the following reasons:

Referral uptake

Knowledge of who does or does not take-up an ERS referral is imperative for improving the degree

of take-up (11). Whether participant characteristics influence the proportion of ERS uptake is uncertain. It is important to know what referral demographics are consistently reported, or unreported, and how they relate to uptake; to gain an understanding of what and how these are influencing scale of ERS uptake. In particular, it is not clear whether those with certain medical conditions have a greater uptake, and whether socio-economic or demographic characteristics influence an individual's uptake ${ }^{(21)}$. Unless uptake and characteristics of people using ERS are known then it is difficult to interpret the extent of generalizability of effect to groups of the population i.e. understand what works, why and for whom.

\section{Attendance and Adherence}

Slade et al. (22) described adherence as the extent to which a person's behaviour corresponds to the agreed referral. It is important within the context of the present review to distinguish the difference between adherence and attendance. Presently, adherence to ERS refers to the percentage of sessions attended ${ }^{(11,15)}$, which may be more appropriately classified as attendance. Adherence then would refer to the participant's behaviour in completing the prescribed PA. Attendance alone does not signify a suitable level of exercise intensity undertaken to benefit from the prescribed PA. For those who complete ERS, promising results were reported for reduced skinfolds, systolic blood pressure and $\mathrm{BMI}{ }^{(23)}$, greater self-efficacy to overcoming PA barriers ${ }^{(24)}$ and higher self-reported PA levels (25). However, failure to attend and adhere to the prescribed programme reduces the 


\section{A Review of Reviews of Exercise Referral Schemes.}

opportunity a participant has for achieving these benefits. As such, it is important to understand what characteristics influence participants' attendance and adherence levels. A valid and reliable assessment of both attendance and adherence is essential for drawing valid conclusions about ERS (22).

\section{Behaviour Change Techniques}

In evaluating a complex intervention such as ERS, there is a requirement to understand the theoretical underpinning at every stage of the intervention, what it is based upon, and the mechanisms through which behaviour change is achieved $(26,27)$. In the same way that the PA content of ERS schemes varies, and is determined between service provider and participant ${ }^{(28)}$, no single explicit behaviour change theory or technique is embedded within ERS (26). The explicit reporting of the components within a complex intervention such as ERS, including its contextual factors such as BCT's utilised, is imperative in understanding; what facilitates uptake, attendance and adherence to ERS and further implementation of ERS (22).

The current review of reviews aims to systematically collate and evaluate the evidence base of review level findings around ERS. It will address what is reported and the consistent and contradictory observations at both a theoretical and practical level. The review aims to: 1) describe definitions rates and participant characteristics associated with ERS uptake; 2 ) to describe definitions rates and participant characteristics associated with attendance at and adherence to ERS schemes; and 3) to describe theoretically-informed approaches associated with ERS uptake and attendance. Furthermore, this review will establish what key features of ERS reporting go relatively underreported to inform future studies of ERS. This review may lead to a clear consensus positively affecting both research and practice in a critical area that could improve the health and wellbeing of individual's with non-communicable disease referred to ERS. 
A Review of Reviews of Exercise Referral Schemes.

92

93

94

95

96

97

98

99

100

101

102

103

104

105

106

107

108

109

110

111

112

113

114

115

116

\section{Methods}

\section{Data Sources and Search Strategy}

The PRISMA (Preferred Reporting Items for Systematic Reviews and Meta-Analyses) statement guided the conduct of this narrative review of reviews ${ }^{(29)}$. Studies were identified by structured electronic database searches. One author (RP), who is a librarian and information specialist, searched 15 electronic databases (CINHAL, Medline, Embase, Cochrane Library, SportsDiscus, PsychInfo, SCOPUS, HMIC, AMED, Public Health Database, ASSIA, CRD databases, Prospero, Web of Knowledge, Campbell Collaboration Library and PubMed), in November 2016, for systematic reviews and meta-analysis of exercise referral schemes in adults published in English language with no date restriction. Additional reviews were searched until June 2017, via reference lists of included literature, alongside searches of UpTodate, BMJ Best Practice and Dynamed Plus. Search strategies were constructed and then amended and agreed by group consultation of all authors. As an example, search terms used for Medline are available within the supplementary material (Supplementary material 1). The protocol and the purpose of the current study were designed beforehand; however, these were not published or uploaded on the web.

\section{Review Selection.}

Review inclusion and exclusion criteria were constructed by CS and then amended and agreed by group consultation and described in Table 1. One author (CS) independently examined titles and abstracts against the inclusion and exclusion criteria. Three authors (GH, TG \& SG) independently assessed a randomised sub sample of papers, amounting to $20 \%$ of the total title and abstract sample. A group (CS, GH, TG \& SG) consensus conferred on which papers were progressed further to full text review and which excluded. Full text review articles were then obtained and assessed by two authors (CS \& SG) against the inclusion and exclusion criteria described in Table 1. Figure 1 describes the justification of excluding papers at multiple stages of the PRISMA protocol. Any discrepancies were discussed until a consensus was reached. 
A Review of Reviews of Exercise Referral Schemes.

117

118

119

120

121

122

123

126

127

128

131

132

133

134

135

136

137

138

\section{Data Extraction}

Data was extracted by one author (CS) against a data extraction template and verified by one coauthor (SG), with any discrepancies discussed until resolution. Eleven predefined constructs relating to the effectiveness of interventions were used (supplementary material 2), taking guidance from work conducted by Greaves et al. ${ }^{(30)}$.

\section{Grading of Evidence}

Each review was graded independently and empirically in duplicate (by CS \& SG) with any discrepancies discussed. The AMSTAR (A Measurement Tool to Assess Systematic Reviews) criteria was used to grade reviews. AMSTAR grades scores as low quality (0-3), medium (4-7) and high (8-11) (31). While the AMSTAR tool was developed to assess reviews of randomized controlled trials (RCTs) only, it has been shown to be applicable to reviews of non-randomized studies, demonstrating good psychometric properties ${ }^{(32)}$. Cohen's kappa was calculated as a measure of reliability for each AMSTAR item. Kappa values less than 0 were rated as less than chance agreement; $0.01-0.20$, slight agreement; $0.21-0.40$, fair agreement; $0.41-0.60$, moderate agreement; $0.61-0.80$, substantial agreement; and 0.81-0.99, almost perfect agreement ${ }^{(32)}$. Additionally, reviews were graded on the type of evidence they were reviewing, such as randomised controlled trials (see Table 2). The classification of AMSTAR (e.g. 9) was then combined with the type of evidence (e.g. A). For example, high-quality systematic reviews of randomised controlled trials was coded as 9A.

\section{Analysis}

A narrative synthesis of the results indicating the quality of the evidence was more appropriate for a review of reviews and was considered for the following reasons: a lack of consistency within reporting of results to undertake a meta-meta-analysis, and the variety of study interventions examined within the reviews. Furthermore, the present review aimed to understand the "how and why' of ERS in order to gain an insight into the manner that ERS is reported. In accordance with reporting guidelines for systematic reviews, a PRISMA checklist is available for this review. 
A Review of Reviews of Exercise Referral Schemes.

Results

143 A systematic search identified 3211 potentially relevant articles (Figure 1). Following exclusion of 144 duplicates and non-relevant articles, 39 articles were retrieved in full and assessed. One additional 145 article was identified in March 2017 via a database alert that was initially set up from the original 146 database search. Eleven reviews met the pre-established inclusion criteria (Table 1).

\section{Review Characteristics}

148 All eleven reviews reported upon an aspect of effectiveness within ERS: seven reviews examined referral to, uptake and attendance at ERS $(2,9,10,11,12,14,15)$ with eight reporting for whom ERS is appropriate, unique items are reported separately.

\section{Review quality}

The methodological quality of included reviews was generally of a medium standard (median AMSTAR score $=7.50$, mean AMSTAR $=7.00)$. Only three systematic reviews examined evidence adjudged to be of high strength (Table 2). Table 3 illustrates the combined duplicate reviewer AMSTAR scoring and strength of evidence within the reviews. The mean inter-rater reliability 


\section{A Review of Reviews of Exercise Referral Schemes.}

methodological weaknesses adjudged via AMSTAR were, the lack of lists indicating the included and excluded studies and the assessment of potential bias within the selection of articles (e.g. in metaanalysis assessment of publication bias should include a combination of graphical aids such as funnel plot and/or statistical tests such as Egger regression test or Hedges-Olken). The greatest methodological strengths were the comprehensive nature of the literature searches performed and the assessment and documentation of the scientific quality of the included studies. Table 4 highlights the reported uptake and attendance figures across reviews. Some reviews were able to pool results in order to conduct meta-analysis. Other reviews were not able to achieve this due to the lack of consistent reporting within the original papers. Recording and reporting methods of uptake, attendance, adherence and theory varied within reviews and are detailed below.

\section{Narrative of Results}

\section{Referral uptake}

Uptake was defined within three linked reviews as attending the initial consultation $\left.{ }^{111}, 12,14\right)$. Two reviews did not provide a definition for uptake $(10,15)$, while one review indicated that it struggled to define uptake due to differences reported within evaluations ${ }^{(9)}$. Table 4 highlights the reported uptake across reviews; demonstrating a range of uptake values between $35 \%$ and $85 \%$. Three of the five reviews $(9,10,12)$ which report on uptake highlight similar values around $65 \%$. Importantly, no review reported characteristics for participant who failed to take up ERS representing around 30$40 \%$ of participants referred. Where reviews have reported characteristics relating to take-up, these figures are derived from participants present within the scheme.

One high quality review reported that two studies demonstrated women were more likely to takeup ERS than men ${ }^{(12) . ~ H o w e v e r, ~ t w o ~ s t u d i e s ~ w i t h i n ~ t h a t ~ r e v i e w ~ s h o w e d ~ n o ~ a s s o c i a t i o n ~ b e t w e e n ~}$ gender and uptake of ERS. Pavey et al. (11) concluded that being female and of increasing age was a positive predictor of uptake. A low quality review summarised that uptake was greater within females $\left(60-40 \%\right.$ for females vs. males) ${ }^{(9)}$. Their review importantly highlighted that reporting of 


\section{A Review of Reviews of Exercise Referral Schemes.}

characteristics was poor and only reported within five primary studies ${ }^{(9)}$. Pavey et al. ${ }^{(11)}$ reported that participants who were more deprived and suffered from a respiratory diagnosis were more likely to take-up ERS than those with the same diagnoses who were least deprived (OR $1.45,95 \% \mathrm{Cl}$ 1.06 to $1.99, p<0.05)$. A high quality review reported that pre-existing medical diagnoses were not separately reported which prevented conclusions being drawn within these subgroups ${ }^{(13)}$. One medium quality review reported that participants referred with mental health problems were more likely to participate in ERS than those with no medical referral (OR 1.79, 95\% $\mathrm{Cl} 1.24$ to $2.39, \mathrm{p}<0.01$ ) ${ }^{(11)}$. However, participants with cardiovascular disease were more likely to engage than participants with mental health problems (OR $0.33,95 \% \mathrm{Cl} 0.27$ to $0.57, \mathrm{p}<0.01$ ), musculoskeletal problems (OR $0.75,95 \% \mathrm{Cl} 0.58$ to $0.99, \mathrm{p}<0.05)$, or overweight/obesity problems (OR $0.63,95 \% \mathrm{Cl} 0.50$ to 0.81 , $\mathrm{p}<0.01)$. Overweight or obese participants were more likely to take-up ERS than smokers ${ }^{(11)}$. Referral uptake to ERS was greater for participants referred from a cardiac nurse over those referred from either a general practitioner or practice nurse ${ }^{(11)}$.

\section{Attendance and Adherence}

206 The most important finding from the reviews, was a lack of reporting on exercise prescription 207 adherence. No review detailed the type of exercise prescribed or the extent to which the participant 208 adhered to the prescription. Reviews did report the term adherence, however, it must be understood that this term was a reference to attendance at the programme. As such, the term adherence from the original reviews is replaced by the more appropriate term of attendance in the

211 current manuscript. Large inconsistences were observed surrounding attendance figures. Four 212 studies failed to define attendance by determining a threshold, instead acknowledging the term as, 213 'duration of participation within ERS' $(9,10,14,15)$. Pavey and colleagues used a threshold of $\geq 75 \%$ 214 attendance of available sessions within an ERS programmes as its inclusion criteria for studies ${ }^{(11,12) .}$ 215 Objective measures, such as the use of leisure centre records, were underutilised (9). 


\section{A Review of Reviews of Exercise Referral Schemes.}

216

217

218

219

220

221

One high quality and one medium quality review, based upon the same initial systematic search, reported that males were more likely to attend from two studies, while three further studies within the reviews found no such association with attendance ${ }^{(11,12)}$. A further low quality review reported attendance was higher in males ${ }^{(9)}$. Their review did report one primary study citing higher attendance in women and two RCT's finding no relationship between sexes ${ }^{(9)}$. Increasing age was positively associated with attendance to ERS from five studies in one review, however, it additionally reported two studies suggesting no such relationship (11). Gidlow et al. ${ }^{(9)}$ reported two evaluations indicating increasing age and being retired were associated with better attendance. However, increasing age was found to reduce participation in PA from one RCT and one evaluation, while one $\mathrm{RCT}$ reported no relationship ${ }^{(9)}$. One medium quality review adds to these mixed views surrounding increasing age by reporting that attendance appeared to be higher in older participants (16). Increasing attendance was more prevalent in participants who had higher baseline activity levels and were overweight ${ }^{(16)}$. Gidlow et al. ${ }^{(9)}$ reported one study, which indicated that socioeconomic characteristics were unrelated to attendance. One high and one medium quality review indicated that socioeconomic characteristics were poorly reported, preventing any clear conclusions on the impact of different socioeconomic characteristics ${ }^{(16,18)}$.

One review acknowledged that a medical diagnosis was a factor that could affect attendance, however they reported it was not consistently demonstrated throughout their included studies ${ }^{(9)}$. Participants with cardiovascular disease were more likely to attend than participants with pulmonary disease ${ }^{(11)}$. Physical health problems were a greater predictor of attendance than mental health issues $(22 \%$ vs $34 \%, p<0.001)$, however it was not reported what was defined as a physical health problem ${ }^{(11)}$. Diabetic participants were less likely to attend ERS (OR $0.76,95 \% \mathrm{Cl} 0.63$ to 0.93 , $\mathrm{p}<0.01)$ than those with cardiovascular disease ${ }^{(11)}$. Conversely, a medium quality review reported that participants referred with sedentary lifestyles or diabetes demonstrated a higher adherence than those with cardiovascular disease or obesity ${ }^{(15)}$. A high quality review reported no consistent 


\section{A Review of Reviews of Exercise Referral Schemes.}

241 difference in attendance rates between participants within one of three at-risk groups: smoking, 242 obesity and hypertension ${ }^{(14)}$.

243 One high quality review identified seventeen studies which highlighted peer support and supervision 244 support from staff as a facilitator for attendance to the referral programme ${ }^{(2)}$. Barriers to 245 attendance were noted as: the location (distance to travel); difficulties reaching the activities by 246 public transport; perceived safety of the location; timings of sessions; and cost ${ }^{(2)}$. A medium quality 247 review indicated that European programmes had greater attendance over American, Australian or 248 Canadian programmes ${ }^{(15)}$. A meta-regression shows the duration of an ERS programme is not 249 correlated to the attendance rate ${ }^{(15)}$. However, a follow up period greater than six months, after the 250 cessation of the programme, did have a positive effect on attendance when the scheme was running 251 (15)

\section{Behaviour Change Techniques}

High quality evidence from two reviews of randomised controlled trials ${ }^{(13,18)}$ showed that the transtheoretical model of behaviour change was the most frequently utilised underlying theory of intervention design, while social cognitive theory, theory of planned behaviour, and health belief model were used less. One low quality review ${ }^{(19)}$ that did not directly report on any theoretical underpinnings, suggested that schemes should consider implementing theory driven approaches to behaviour change. One medium ${ }^{(10)}$ and one high ${ }^{(11)}$ quality review commented that the greatest challenge for ERS was in increasing uptake and improving attendance ${ }^{(10,11)}$. One review reported directly on how attendance were affected by theoretical techniques ${ }^{(16)}$. Four reviews reported on

261 theoretical techniques to increase PA time or clinical markers $(12,13,14,18)$. With the exception of a few 262 primary studies, reviews reported little reference to the delivery, fidelity, or BCT's of health 263 behaviour change $(10,12,13,14,18)$. Motivational interviewing was the most frequently utilised $B C T$, cited 264 within eight reviews $(9,10,12,13,14,16,18,19)$, with only one review indicating the fidelity of the technique 265 (14). One medium quality review reporting upon on a primary study, report that participants who 


\section{A Review of Reviews of Exercise Referral Schemes.}

266

267

268

269

270

271

received motivational interviewing combined with free vouchers had a greater self-reported physical activity score at 12 weeks, than a control group given advice alone (all groups combined, $\mathrm{P}<0.001$ ) (16). The greatest increase in PA was observed in the intensive motivational interviewing and free vouchers group which offered six motivational interviewing sessions $(55 \%, \mathrm{P}<0.001)$. No difference was evident at one year post-intervention between groups ${ }^{(16)}$. Morgan ${ }^{(16)}$ further reported that exercise-plus-motivation vs exercise-only elicited a greater attendance over a 20 week programme $(62 \%$ vs $38 \%, \mathrm{P}<0.05)$. A further primary study reported that there was no significant difference between three groups attending sessions when comparing, exercise alone, instructions based on the 'relapse prevention' model, or sessions that included re-enforcement methods (prizes for high attendance). Attendance attrition was reported at 30\% and $72 \%$ for all groups at 9 and 18 weeks, respectively ${ }^{(16)}$. One high quality review indicated that motivational interviewing was utilised predominantly by different practitioners but provides no indication of its impact ${ }^{(18)}$. A high quality review reported two contrasting studies; one indicated that attendance was positively influenced by participant levels of self-determination, and one study found no association between these parameters ${ }^{(12)}$. A medium quality review reported participant dissatisfaction when lacking social support, or with an exercise leader lacking motivational skills ${ }^{(10)}$.

\section{Discussion}

This review is the first to systematically identify, collate, and grade the evidence from review level findings on ERS. The creation of this review highlights and summarises the consistent and contradictory findings surrounding the reporting of: 1) uptake to ERS; 2) attendance and adherence to ERS; and 3) BCT's relating to attendance and adherence. We discuss the characteristics of studies and definitions used within them and how they affect uptake, attendance and adherence. We highlight how a lack of reporting and knowledge of what is delivered within ERS limits our insight into both attendance rates and adherence to the prescribed programme. Where reported, considerations are made as to why participants with certain characteristics (e.g. medical referral) are more likely to take up and attend schemes. Lastly, we discuss the reporting of theoretical constructs 
A Review of Reviews of Exercise Referral Schemes.

292

and BCT's, proposing reporting tools that could be considered to help improve uptake and attendance.

\section{Referral uptake}

295

Historically, through the reviews, it is reported that uptake of ERS sits around $60-70 \%$ of those

referred. This suggests that no improvements have been made in increasing the number of participants starting a scheme over the years examined. Uptake to ERS was greater in randomised controlled trials than observational evaluations, as reported in Table 4. Explanation for this could centre on participants offering full consent, coupled with more stringent recruitment processes often excluding participants, which might have elicited higher commitment to the programme ${ }^{(33,34)}$. Importantly, no review reported detailed characteristics of participants who are referred, but fail to make contact with an ERS. Instead, reviews have commented upon uptake figures from those who start a scheme. While this review cannot comment on the research priorities of previous reviews or individual studies, we suggest that this reflects a crucial gap in understanding within the primary data. It is important to this review to question why this gap exists, because if large sub groups of the population are not taking up a referral they cannot benefit from the programme.

Reviews have attempted to unpick participant referral characteristics, for example, there have been suggestions that participants referred for a medical reason may show greater referral uptake compared to those with no specific referral ${ }^{(11)}$, however, most reviews are less clear on such a hierarchy of take up. Referral to ERS from a cardiac nurse appears more effective than referral from any other health professional ${ }^{(11)}$, from which it could be inferred that the hospitalisation 312 surrounding the condition has served as a teachable moment. 'Teachable moments' have been 313 described within health behaviour as a noticeable feature of a patient's circumstance which prompts 314 a change of behaviour ${ }^{(35)}$. These opportunities are utilised within the patient-clinician interaction or by patients alone to foster positive health behaviours, such as increasing physical activity levels ${ }^{(35,}$ 
A Review of Reviews of Exercise Referral Schemes.

317 Systemic features, such as the expectations of those involved, communication skills of the health practitioner, and medical practice setting will influence the situation ${ }^{(36)}$.

319 Reviews have indicated participant profiling is varied and this lack of information on subgroups prevents any inference being drawn about who ERS is best suited for. Pampel et al. ${ }^{(37)}$ comment that low socioeconomic status groups have fewer opportunities to engage in services that promote good health, yet the picture is not clear in ERS. For example, Pavey et al. ${ }^{(11)}$ have shown participants who had a medical referral coupled with a low socioeconomic status were more likely to take up ERS over participants with a higher economic status, but this was only within participants with a respiratory diagnosis. Further reviews ${ }^{(16,18)}$ suggest that insufficient reporting of socioeconomic status within ERS prevents any conclusions being reached.. The complexity of uptake of ERS, insufficient reporting within ERS $(16,18)$ coupled with a lack of clear guidance within the socioeconomic and health behaviour literature ${ }^{(37)}$ means it is very difficult to produce generalizable statements surrounding the socioeconomic status and take up of ERS.

\section{Adherence and Attendance}

In contrast to uptake, attendance figures across reviews appear greater within observational studies. Interpretation of attendance and completion rates should be treated cautiously due to a lack of a standardised protocol (i.e. objective, standardised, universally consistent measure of attendance) for reporting these figures across the literature. The lack of a standardised measure-surrounding attendance could see participants being classified as completing a scheme ${ }^{(12)}$, while the reality may be attendance at a couple of sessions and attendance at the final exit session of the scheme. This invites questions as to why there is no standardized use of an objective count for attendance, since all outcomes of ERS are dependent upon attendance at, and adherence to the programme ${ }^{(9)}$. The review by Pavey et al. ${ }^{(11)}$ concluded that the number of exercise sessions made available within a

340 scheme might elicit higher rates of attendance. Meanwhile Arsenijevic and Groot ${ }^{(15)}$ reported that attendance rates were not correlated to the duration of the programme. Additionally, they report 
A Review of Reviews of Exercise Referral Schemes.

342

that a prolonged follow up with increased contact time with researchers and exercise practitioners post ERS could offer explanations to why attendance was greater during the programme ${ }^{(15)}$. This in itself is suggestive that observational studies may reflect the true nature of ERS (11).

While uptake to ERS was reported to be slightly higher in females, overall, males were more likely to participate within a programme $(9,11,12)$. However, various primary studies within these reviews found no association between gender and attendance. This review questions why there is such a poor base level of reporting with only three reviews, of which two reviews are based upon the same study search criteria $(11,12)$, reporting on gender characteristics and referral uptake $(9,11,12)$. The present review does highlight a greater number of reviews reporting upon medical conditions and the impact they have upon attendance. Despite this greater breadth of reporting, no clear consensus can be reached, with reviews reporting no difference in attendance between at risk groups (14); either participants with cardiovascular disease were more likely to attend (11); or conversely participants with cardiovascular disease were less likely to attend ${ }^{(15)}$.

Inference on reporting of attendance figures could be suggestive of at least two aspects of ERS. First, it could suggest a referral process where the wrong participants are being referred into the system. Resolution of this could be gained through a clearer and more robust understanding of who is referred to ERS ${ }^{(9)}$. Second, it could reflect a lack of knowledge of what participants adhere to within the programme. With no understanding of what is delivered within a programme, from an exercise prescription standpoint, and no reporting on the extent to which individuals adhere to the prescription, few conclusions can be drawn about the effectiveness of the scheme. It is important to question why this gap exists, because if sub groups of the population are not completing the prescribed programme then they clearly cannot benefit from the programme. It is important to acknowledge that participants will stand to benefit from any episode of PA completed, regardless of meeting the prescribed dose. However, it is important to know and understand the frequency, intensity, type, and time (FITT) of prescribed exercise in these programmes, but this is not commonly 
A Review of Reviews of Exercise Referral Schemes.

367

368

369

370

371

372

recorded or reported. Knowledge of the prescribed dose could help to understand if it is too demanding, thus leading to participant drop out, or insufficiently demanding to engage participants or provide a clinical benefit. Development of the Consensus on Exercise Reporting Template allows detailed and explicit reporting of the delivery of ERS for policy makers and practitioners alike (22). Implementation of the Consensus on Exercise Reporting Template will further enhance the knowledge base and understanding for whom prescribed exercise is beneficial. Ultimately, it must be understood that both attendance and adherence are multidimensional constructs affected by the relationship between participant and practitioner intertwined with participant centric factors (e.g. mood state, self-efficacy, time and forgetfulness) ${ }^{(22)}$ and potentially exercise prescription centric factors (e.g. frequency, intensity, time and type of prescription).

\section{Behaviour Change Techniques}

Development of ERS was based upon policy recommendations rather than theoretical guidelines developed for health promotion interventions ${ }^{(15)}$. Riddoch, Puig-Riberia and Cooper ${ }^{(17)}$ reported that early ERS's were not based upon any behaviour change model. Failure to acknowledge, deliver or evaluate behaviour change models during a programme prevents clear evaluation of the ERS. More often, the end point (e.g. PA levels, blood glucose concentration, attendance and adherence levels) is the result of behaviour change. Evaluating and reporting interventions on outcomes alone is problematic with an array of influences that could determine the path between behaviour and outcome ${ }^{(38)}$. This is evident within the literature where success of ERS is judged as the outcome evaluation without taking into consideration the methods underpinning it, such as BCT utilised to motivate uptake or increase attendance levels to ERS or the quality of those delivering these BCT's.

Theories of behaviour change provide a validated framework to understand not only how behaviour changed, but importantly why behaviour changed ${ }^{(39)}$. A review by Dombrowski et al. ${ }^{(39)}$ found that the delivery and features of behaviour change techniques was heterogeneous in nature. Additionally, a later review reported $44 \%$ of interventions did not report use of a theoretical 


\section{A Review of Reviews of Exercise Referral Schemes.}

392

framework ${ }^{(40)}$. This lack of reporting within both reviews of ERS and the wider field of health behaviour prevents any clear guidance upon which theory or BCT, or how it is operationalized, is most effective to understand health behaviour, and health behaviour change (40).

In the present review we have highlighted how uptake of ERS and attendance at ERS could be influenced by participant characteristics or external factors. Whilst factors such as that of socioeconomic status and teachable moments, have been discussed, a further consideration would be the theoretical perceived risk. The health belief model and the common-sense model hypothesise that perceived severity combined with perceived susceptibility and external cues to actions are strong contributing factors within an individual's perception of threat ${ }^{(41,42)}$. While our review cannot comment upon direct causality to why individuals with certain medical conditions have a greater uptake or attendance rate, we can stress the importance of trying to understand these factors. This understanding comes from the ability to record, report and evaluate these perceptions. Tools such as the Illness Perception Questionnaire - allow for a greater understanding of what an individual perceives of their condition ${ }^{(43)}$. The brief Illness Perception Questionnaire has been shown as a valid and reliable measure of illness perception distinguishing between various illness groups and importantly for ERS, is a predictor of attendance at cardiac rehabilitation classes ${ }^{(43)}$. Incorporation of tools such as the Illness Perception Questionnaire seem essential to trying to understand theoretical factors or BCT's impacting upon ERS uptake or attendance.

In line with Prestwich et al. ${ }^{(40)}$, we report that the transtheoretical model of behaviour change and social cognitive theory, alongside BCT's such as motivational interviewing, are used, but ultimately are underutilised tools. Fundamentally, the lack of theoretical reporting within ERS generates more questions than it solves. Is the lack of reporting due to there being a lack of delivery or, more an inability to efficiently test and report its delivery? Without assessing any possible association between the $\mathrm{BCT}^{\prime} \mathrm{s}$ in conjunction with the theory they are based within, we are unable to further our understanding of possible effects ${ }^{(39)}$. The reporting of an intervention's BCT and delivery (e.g. 


\section{A Review of Reviews of Exercise Referral Schemes.}

417

418

419

420

421

422

423

424

426

427

428

duration, intensity, setting, group vs individual, verbal vs written and the skill level of the practitioner deliverers) ${ }^{(39)}$ is imperative if we are to understand which techniques are appropriate within ERS for improving uptake and attendance.

\section{Implications for practice and policy}

This review highlights the need for consistent reporting methods to be implemented within ERS. The ability to robustly detail participant characteristics from the point of referral to exiting the scheme (at any stage) will only enhance the understanding of the ERS process. The ability to gain an understanding of what is being delivered at a theoretical level (e.g. BCT's, such as goal setting or relapse prevention and the delivery process of these BCT's) and physical activity level (e.g. frequency, intensity, type and time) would allow strong associations to be attributed, or not, to these features, notably for understanding levels of attendance and adherence. Failure to have these fundamentals in order places increased pressure upon ERS, notably when the National Institute for Health and Care Excellence ${ }^{(4)}$ are highlighting the scarce evidence of effectiveness for ERS schemes. The use of tools such as the Consensus on Exercise Reporting Template, Illness Perception Questionnaire or behaviour change taxonomies will further help to understand how and why behaviour changed within programmes. Greater understanding at any level will benefit stakeholders, health practitioners and participants alike, by providing individualised care pathways, in achieving the most effective results from stretched resources and improving programme implementation and viability.

\section{Strengths and limitations.}

This is the first systematic review of reviews focusing on ERS. By taking an overarching view of all the reviews we have been able to highlight key areas that require exploration to inform future evaluation of ERS. However, due to the nature of reviewing systematic reviews, we are unable to explore and provide detailed in-depth mechanistic knowledge surrounding ERS effectiveness. Additional problems arise for evidence reporting namely around AMSTAR grading. While AMSTAR 


\section{A Review of Reviews of Exercise Referral Schemes.}

442 has been shown to demonstrate good psychometric properties, it was developed after four of the 443 included reviews had been published. Subsequently two of these reviews were classified as low 444 quality yet they provide vital insights into ERS. Whilst this review of reviews examined 11 systematic 445 reviews, which in turn sourced 221 citations, $44 \%$ of citations were duplicated across reviews 446 reducing the breadth of data available. While this review draws attention to tools such as the IIIness 447 Perception Questionnaire to assess a participant's perception towards their illness, this review is 448 unable to address participant's perspectives towards ERS. This is impart due to the scope of this 449 review. We acknowledge primary studies that have provided qualitative insight towards participant 450 perspectives of ERS ${ }^{(3,44)}$; however, there exists a knowledge gap in utilising this for implementation 451 refinement of ERS.

\section{Directions for future research}

453 Research should work hand-in-hand with practice and policy makers, in the first instance, to 454 facilitate robust participant profiling: 1 ) to capture data on individuals referred but not taking up the 455 referral to help reduce health inequalities; 2 ) to generate a consensus on monitoring attendance and 456 adherence to ERS with objective measures for reporting it; and 3) to incorporate explicit reporting of 457 BCT's and what they are being used for. With a consistent and coherent basis for reporting, future evaluations and RCT interventions will be able to explore a multitude of potential interventions, safe in the knowledge they are grounded within consistent reporting, allowing for direct comparison between schemes. Greater research is needed surrounding ERS programme content. The very nature of ERS referral is for participants with lifestyle disease, yet only small to moderate clinical improvements have been shown within the literature. The use of tools such as the Consensus on Exercise Reporting Template will help drive research on frequency, intensity, and type of PA delivered within programmes. Tools such as the Illness Perception Questionnaire have the potential to build greater understanding of whether individuals are potential candidates for referral to ERS or not and perhaps leading to a more efficient uptake process. These tools may help in understanding

467 who and what specifically, contributes to each part of the complexity surrounding ERS. Should 


\section{A Review of Reviews of Exercise Referral Schemes.}

468

469

470

471

472

473

interventions be focused upon deliverers, the content and its delivery methods within the scheme; or would research be better utilised in understanding behaviour change towards ERS. Further research is required to establish methods that address the challenging nature of measuring $B C T$ within ecological valid environments, such as ERS. Other research may be able to track participants over the long-term, reporting on future primary care or hospital admissions with the ability to link it back to ERS data. While the potential for future research is hypothesised here, it is fundamentally reliant on robust, clear, standardised and explicit reporting.

\section{Conclusions}

Exercise referral schemes benefit from not being constrained within a rigid framework, allowing a varied spectrum of delivery. However, it would appear this currently may also be detrimental in understanding what works, why and for whom. Complex interventions such as ERS, which involves multiple input from various health practitioners, requires robust and consistent recording and reporting of all its facets at every stage of the process. ERS outcomes which are often judged in terms of increased physical activity, are subject to multiple, separate and complex constructs along the way. We have reported that uptake levels to schemes have not changed and are potentially influenced by a variety of participant characteristics. Importantly, there is a requirement to know who the people are that are not taking up referral and thus missing the opportunity to potentially benefit from ERS. We have identified that attendance within schemes is potentially influenced by both participant characteristics and scheme definition. Critically there is no knowledge or reporting to-date on what participants adhere to within ERS. We have also highlighted that BCT's are poorly reported, preventing any knowledge of how and why change may have occurred. Failure to robustly produce effective reporting methods or have a clear scheme wide consensus prevents any firm conclusions on causal effect. Ultimately, a lack of reporting prevents any research from accurately validating its hypothesis ${ }^{(45)}$ and within the construct of this review, assigning a true reflection on the effectiveness of ERS. 
A Review of Reviews of Exercise Referral Schemes.

493

494

495

496

497

498

499

500

501

502

503

504

505

506

507

508

509

510

511

512

513

514

515

516

517
1. Department of Health, Physical Activity and Health Improvement and Protection. Start Active, Stay Active: A report on physical activity from the four home countries' Chief Medical Officers. London: Department of Health. 2011.

2. Morgan F, Battersby A, Weightmam AL, Searchfield L, Turley R, Morgan H, Jagroo J, Ellis S. Adherence to exercise referral schemes by participants - what do providers and commissioners need to know? A systematic review of barriers and facilitators. BMC Public Health. 2016;16(227):1-11.

3. Din NU, Moore GF, Murphy S, Wilkinson C, Williams NH. Health professionals' perspectives on exercise referral and physical activity promotion in primary care: Findings from a process evaluation of the National Exercise Referral Scheme in Wales. Health Education Journal. 2015;74(6):743-757.

4. National Institute for Health and Care Excellence. Physical Activity: exercise referral schemes. Public Health Guideline. Manchester: NICE. 2014.

5. World Health Organisation. Global recommendations on physical activity for health. Switzerland: WHO Library Cataloguing-in-Publication. 2010.

6. National Institute for Health and Care Excellence. Physical Activity: walking and cycling. Public Health Guideline. Manchester: NICE. 2012.

7. Moore GF, Moore L, Murphy S. Facilitating adherence to physical activity: exercise professionals' experiences of the National Exercise Referral Scheme in Wales. A qualitative study. BMC Public Health. 2011;11(935):1-12.

8. Ward M. Experiences in physical activity promotion in health care settings for primary prevention in the UK. Swiss Sports \& Exercise Medicine. 2014;62(2):26-28.

9. Gidlow C, Johnston LH, Crone D, James D. Attendance of exercise referral schemes in the UK: A systematic review. Health Education Journal. 2005;64(2):168-186. 
A Review of Reviews of Exercise Referral Schemes.

10. Williams NH, Hendry M, France B, Lewis R, Wilkinson C. Effectiveness of exercise-referral schemes to promote physical activity in adults: systematic review. British Journal of General Practice. 2007;57(545):979-986.

11. Pavey T, Taylor A, Hillsdon M, Fox K, Campbell J, Foster C, Moxham T, Mutrie N, Searle J, Taylor R. Levels and predictors of exercise referral scheme uptake and adherence: A systematic review. Journal of epidemiology and community health. 2012;66(8):737-744.

12. Pavey T, Anokye N, Taylor A, Trueman P, Moxham T, Fox K, Hillsdon M, Green C, Campbell J, Foster C, Mutrie N, Searle J, Taylor R. The clinical effectiveness and costeffectiveness of exercise referral schemes: a systematic review and economic evaluation. Health Technology Assessment. 2011;15(24):1-270.

13. Pavey TG, Taylor AH, Fox KR, Hillsdon M, Anokye N, Campbell JL, Foster C, Green C, Moxham T, Mutrie N, Searle J, Trueman P, Taylor RS. Effect of exercise referral schemes in primary care on physical activity and improving health outcomes: Systematic review and meta-analysis. BMJ open. 2011;343:1-14.

14. Campbell F, Holmes M, Everson-Hock E, Davis S, Buckley Woods H, Anokye N, Tappenden P, Kaltenthaler, E. A systematic review and economic evaluation of exercise referral schemes in primary care: a short report. Health Technology Assessment. 2015;19(60):1-105.

15. Arsenijevic J, Groot W. Physical activity on prescription schemes (PARS): do programme characteristics influence effectiveness? Results of a systematic review and metaanalyses. BMJ Open. 2017:1-13.

16. Morgan O. Approaches to increase physical activity: reviewing the evidence for exercisereferral schemes. Public Health. 2005;119:361-370.

17. Riddoch C, Puig-Riberia A, Cooper A. Effectiveness of physical activity promotion schemes in primary care: a review. London: Health Education Authority. 1998. 
A Review of Reviews of Exercise Referral Schemes.

18. Orrow G, Kinmonth AL, Sanderson S. Sutton S. Effectiveness of physical activity promotion based in primary care: systematic review and meta-analysis of randomised controlled trials. BMJ Open. 2012;344:1-17.

19. Sorensen JB, Skovgaard T, Puggaard L. Exercise on prescription in general practice: A systematic review. Scandinavian journal of primary health care. 2006;24(2):69-74.

20. Jones $F$, Harris $P$, Waller $H$, Coggins $A$. Adherence to an exercise prescription scheme: The role of expectations, self-efficacy, stage of change and psychological well-being. British Journal of Health Psychology. 2005;10:359-378.

21. Dinan S, Lenihan P, Tenn T, lliffe S. Is the promotion of physical activity in vulnerable older people feasible and effective in general practice? British Journal of General Practice. 2006:791-793.

22. Slade SC, Dionne CE, Underwood M Buchbinder R. Consensus on exercise reporting template (CERT): Explanation and elaboration statement. British Journal of Sports Medicine. 2016;50:1428-1437.

23. Taylor AH, Doust J, Webborn. Randomised controlled trial to examine the effects of a GP exercise referral programme in Hailsham, East Sussex,on modifiable coronary heart disease risk factors. Journal of Epidemiology and Community Health. 1998;52:595-601.

24. Edmunds J, Ntoumanis N, Duda JL. Adherence and well-being in overweight and obese patients referred to an exercise on prescription scheme: A self-determination theory perspective. Psychology of Sport and Exercise. 2007;8:722-740.

25. Leijon ME, Bendtsen $P$, Nilsen $P$, Festin $K$, Stahle A. Does a physical activity referral scheme improve the physical activity among routine, primary health care patients? Scandinavian Journal of Medicine \& Science in Sports. 2009;19(5):627-636.

26. Littlecott H, Moore GF, Moore L, Murphy S. Psychosocial mediators of change in physical activity in the Welsh national exercise referral scheme: secondary analysis of a 
A Review of Reviews of Exercise Referral Schemes.

568

569

570

571

572

573

574

575

576

577

578

579

580

581

582

583

584

585

586

587

588

589

590

591

592

593 randomised controlled trial. International Journal of Behavioural Nutrition and Physical Activity. 2014;11(109):1-11.

27. Moore GF, Audrey S, Barker M, Bond L, Bonell C, Cooper C, Hardeman W, Moore L, O'Cathain A, Tinati T, Wright D, Baird J. Process evaluation in complex public health intervention studies: the need for guidance. Journal of Epidemiology and Community Health. 2014;68(2): p.1-2.

28. Woods C, McCaffrey N, Furlong B, Fitzsimons-D’Arcy L, Murphy M, Harrison M, Glynn L, O’Riordan J, O’Neill B, Jennings S, Peppard C. The National Exercise Referral Framework. Health and Wellbeing Division, Health Service Executive. Dublin: Health Service Executive. 2016.

29. Moher D, Liberati A, Tetzlaff J, Altman DG, The PRISMA Group. Preferred reporting items for systematic reviews and meta-analyses: The PRISMA Statement. PLoS Medicine. 2009;6(7):1-6.

30. Greaves CJ, Sheppard KE, Abraham C, Hardeman W, Roden M, Evans PH, Schwarz P. Systematic review of reviews of intervention components associated with increased effectiveness in dietary and physical activity interventions. BMC Public Health. 2011;11(119):1-12.

31. Shea BJ, Grimshaw JM, Wells GA, Boers M, Andersson N, Hamel, C, Porter AC, Tugwell P, Moher D, Bouter LM. Development of AMSTAR: a measurement tool to assess the methodological quality of systematic reviews. BMC Medical Research Methodology, 2007;7(10):1-7.

32. Pieper D, Mathes T, Eikermann M. Can AMSTAR also be applied to systematic reviews of non-randomized studies? BMC Research Notes. 2014;7(609):1-6.

33. Martin KA, Sinden AR. Who will stay and who will go? A review of older adults' adherence to a randomized controlled trials of exercise. Journal of Ageing and Physical Activity. 2001;9:91-114. 
A Review of Reviews of Exercise Referral Schemes.

594

595

596

597

598

599

600

601

602

603

604

605

606

607

608

609

610

611

612

613

614

615

616

617

618
34. Benson K, Hartz AJ. A comparison of observational studies and randomized controlled trials. The New England Journal of Medicine. 2000;345(25):1878-1886.

35. Flocke S, Clark E, Antognoli E, Mason MJ, Lawson PJ, Smith S, Cohen DJ. Teachable moments for health behaviour change and intermediate patient outcomes. Patient Education and Counselling. 2014;96(1):1-16.

36. Lawson PJ, Flocke S, Teachable moments for health behaviour change: a concept analysis. Patient Education and Counselling. 2009;76(1):25-30.

37. Pampel FC, Krueger PM, Denney JT. Socioeconomic Disparities in Health Behaviours. Annual Review of Sociology. 2010;36:349-370.

38. Michie S, Johnston M. Theories and techniques of behaviour change: Developing a cumulative science of behaviour change, Health Psychology Review. 2012;6(1):1-6.

39. Dombrowski SU, Sniehotta FF, Avenell A, Johnston M, MacLennan G, Araújo-Soares V. Identifying active ingredients in complex behavioural interventions for obese adults with obesity-related co-morbidities or additional risk factors for co-morbidities: a systematic review. Health Psychology Review. 2012;6(1):7-32.

40. Prestwich A, Sniehotta FF, Whittington C, Dombrowski SU, Rogers L, Michie S. Does theory influence the effectiveness of health behaviour interventions? Meta-analysis. Health Psychology, 2014;33(5):465-474.

41. Jones CJ, Smith $\mathrm{H}$, Llewellyn C. Evaluating the effectiveness of health belief model interventions in improving adherence: A systematic review. Health Psychology Review. 2014;8(3):253-269.

42. Leventhal H, Meyer D, Nerenz D. The common sense representation of illness danger. In: Rachman, S., Ed., Contributions to Medical Psychology. 1980;2:17-30.

43. Broadbent E, Petrie, KJ. Main J, Weinman J. The brief illness perception questionnaire. Journal of Psychosomatic Research. 2006;60:631-637. 
A Review of Reviews of Exercise Referral Schemes.

619

620

621

622

623

624

625

626

627 Funding source

628 This work forms part of a PhD thesis with the lead author receiving funding from the University of 629 Stirling as part of the Impact Studentship Scheme. 
A Review of Reviews of Exercise Referral Schemes.

630

Tables

Table 1 Inclusion and Exclusion criteria used in collating Systematic reviews inclusion.

1. Not in English Due to lack of translation facilities, all non-English papers were excluded. language

2. Type of study Systematic reviews (which included RCTs, observational studies, casecontrolled or other quasi-experimental studies within them) and metaanalysis were included for review.

3. Type of Interventions promoting changes in physical activity behaviour via a Intervention direct referral from a health practitioner within primary care to a recognised exercise programme (e.g. local leisure centre, local walking scheme). Interventions initiated from secondary care and beyond were excluded alongside any health screening programmes.

4. Type of Adults (16 years and over) of any gender who had been diagnosed or Participants placed at risk of non-communicable disease (e.g. type 2 diabetes, hypertension, cardiovascular disease) from their primary care practitioner. Interventions where participants were sedentary but had no further risk factors but were used as a control groups where included if reported separately.

5. Outcomes Reviews were selected where the primary outcome measures reported were increase in physical activity (e.g. frequency, intensity) uptake and adherence to schemes, physiological and psychological (e.g. changes in Body Mass Index or Short Form (36) health survey). Outcomes could be measured objectively (e.g. with accelerometers) or by self-reported means (e.g. questionnaires). Where reported the cost effectiveness of 
A Review of Reviews of Exercise Referral Schemes.

schemes was included.

631

Table 2 Classification of strength based upon evidence included within reviews.

B Systematic reviews of individual, non-RCTs, case-control studies, cohort studies, controlled before-and-after (CBA), correlation studies or similar.

C Systematic reviews of both RCTs and non-RCTs, case-control studies, cohort studies, controlled before-and-after (CBA), correlation studies or similar.

632 
A Review of Reviews of Exercise Referral Schemes.

Table 3 Total and mean AMSTAR scoring, classification of strength based upon evidence included within reviews and Cohen's Kappa for inter-rate reliability.

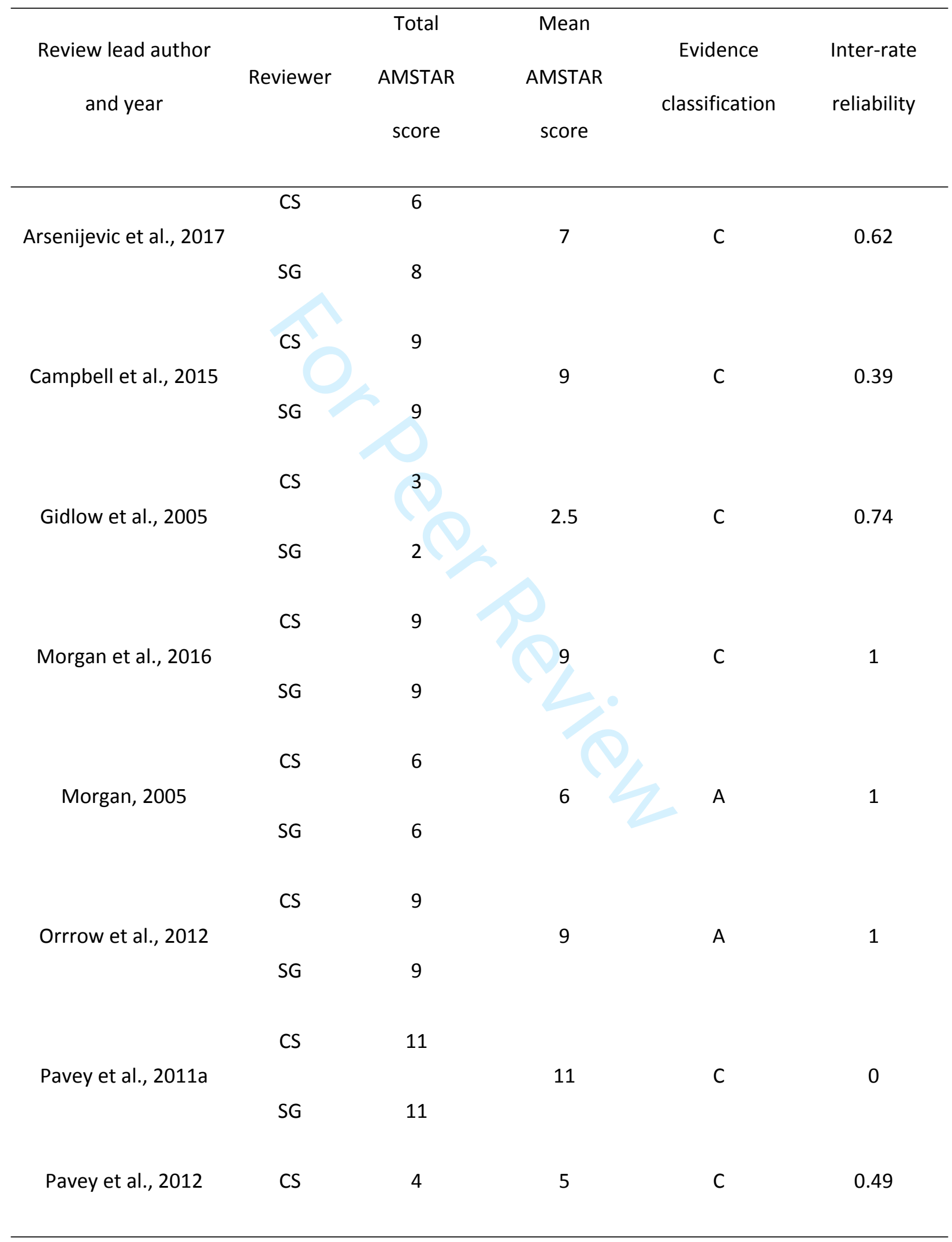


A Review of Reviews of Exercise Referral Schemes.

\begin{tabular}{lccccc}
\hline & SG & 6 & & & \\
Pavey et al., 2011b & CS & 9 & & & \\
& SG & 8 & & & \\
Sorensen et al., 2006 & CS & 2 & 3.5 & C & 0.42 \\
& SG & 5 & & & \\
Williams et al., 2007 & CS & 7 & & C \\
\hline
\end{tabular}

634

Table 4 Reported uptake and attendance figures for Observational studies and RCT's where reported within reviews

\begin{tabular}{|c|c|c|c|}
\hline $\begin{array}{c}\text { Review lead author and } \\
\text { year }\end{array}$ & $\begin{array}{l}\text { Strength of } \\
\text { study }\end{array}$ & Reported uptake & Reported attendance \\
\hline Campbell et al., 2015 & $9 \mathrm{C}$ & $35-85 \%$ & $25-86 \%$ \\
\hline Gidlow et al., 2005 & $2.5 \mathrm{C}$ & $<67 \%$ & $12-25 \%$ \\
\hline \multirow[t]{4}{*}{ Pavey et al., 2011a } & $11 \mathrm{C}$ & Observational studies & Observational studies \\
\hline & & $66 \%(95 \% \mathrm{Cl}=57-75 \%)$ & $49 \%(95 \% \mathrm{Cl}=40-59 \%)$ \\
\hline & & RCTs & RCTs \\
\hline & & $80 \%(95 \% \mathrm{Cl}=61-98 \%)$ & $37 \%(95 \% \mathrm{Cl}=20-54 \%)$ \\
\hline
\end{tabular}


A Review of Reviews of Exercise Referral Schemes.

\begin{tabular}{|c|c|c|c|}
\hline \multirow[t]{4}{*}{ Pavey et al., 2012} & $5 C$ & Observational studies & Observational studies \\
\hline & & $66 \%(95 \% \mathrm{Cl}=57-75 \%)$ & $43 \%(95 \% \mathrm{Cl}=32-54 \%)$ \\
\hline & & RCTs & RCTs \\
\hline & & $81 \%(95 \% \mathrm{Cl}=68-94 \%)$ & $80 \%(95 \% \mathrm{Cl}=61-98 \%)$ \\
\hline Williams et al., 2007 & $7 C$ & $66 \%$ & $12-42 \%$ \\
\hline
\end{tabular}


A Review of Reviews of Exercise Referral Schemes.
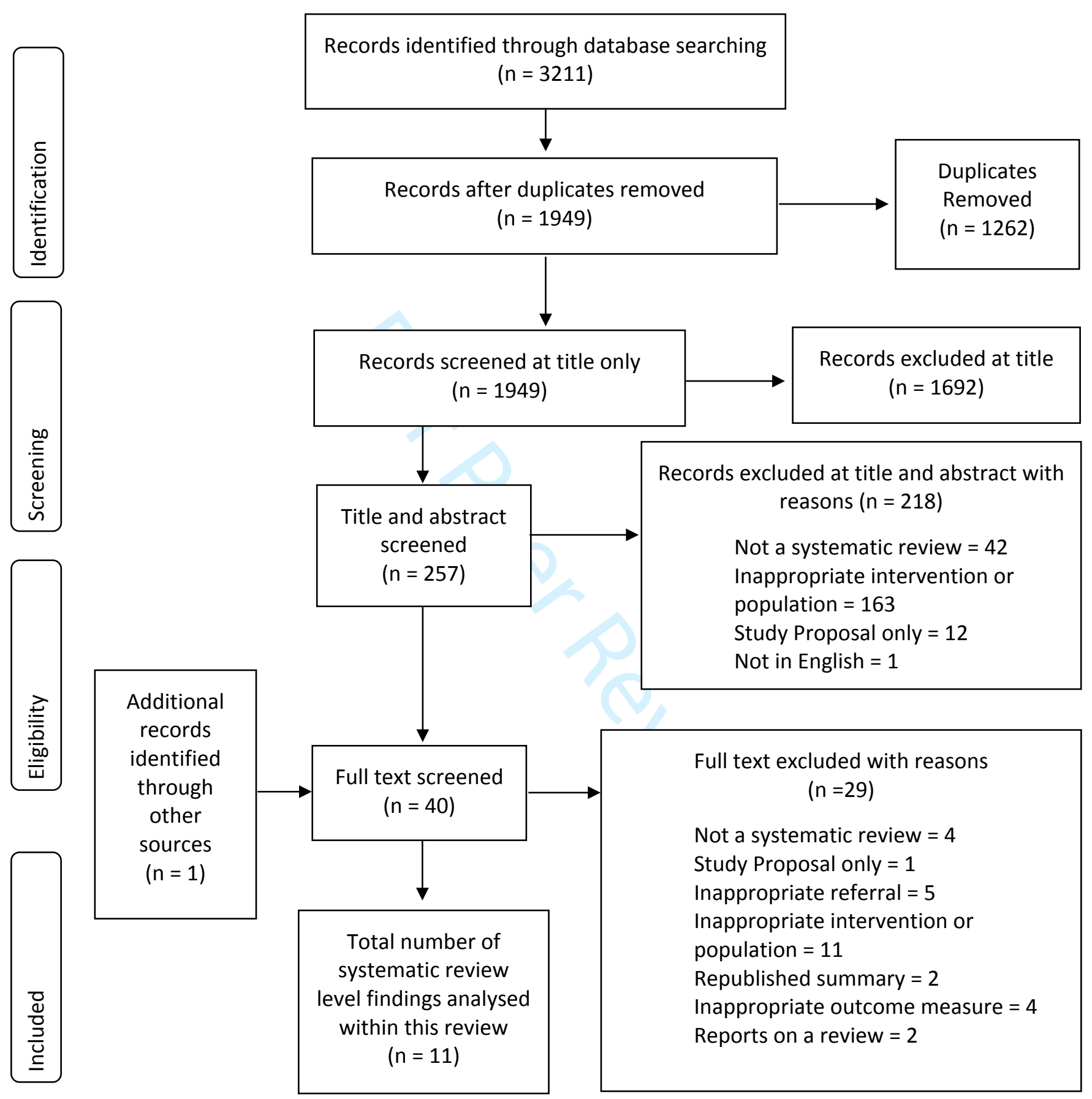

635

Figure 1. PRISMA flow diagram depicting study selection, screening, eligibility for inclusion and analysis. 


\section{Supplementary data.}

Supplementary data 1: Search strategy terms and protocol steps used within Medline database

\begin{tabular}{|c|c|}
\hline Step & Search term \\
\hline 1 & exp Exercise/ \\
\hline 2 & exp Exercise Movement Techniques/ or exp Exercise Therapy/ \\
\hline 3 & exp Sports/ \\
\hline 4 & exp Motor Activity/ \\
\hline 5 & exp Physical Exertion/ \\
\hline 6 & exercis* refer*.af. \\
\hline 7 & refer* exer*.af. \\
\hline 8 & exerc* prescr*.af. \\
\hline 9 & prescr* exerci*.af. \\
\hline 10 & (activ* refer* or refer* activ*).af. \\
\hline 11 & (presc* activ* or activ* precri*).af. \\
\hline 12 & 6 or 7 or 8 or 9 or 10 or 11 \\
\hline 13 & exp Prescriptions/ \\
\hline 14 & (prescribing or prescribe or prescriptions).af. \\
\hline 15 & 13 or 14 \\
\hline 16 & or/1-5 \\
\hline 17 & 15 and 16 \\
\hline 18 & 12 or 17 \\
\hline 19 & limit 18 to english language \\
\hline 20 & (metaanal: or meta-anal:).af. \\
\hline 21 & exp Meta-Analysis/ \\
\hline 22 & 20 or 21 \\
\hline 23 & limit 19 to meta analysis \\
\hline 24 & 19 and 22 \\
\hline 25 & 23 or 24 \\
\hline 26 & (systematic* review* or review* systematic*).af. \\
\hline 27 & limit 19 to systematic reviews \\
\hline 28 & 19 and 26 \\
\hline 29 & 27 or 28 \\
\hline 30 & systematic review*.af. \\
\hline 31 & 19 and 30 \\
\hline 32 & 27 and 31 \\
\hline 33 & 29 not 25 \\
\hline 34 & 25 or 29 or 33 \\
\hline
\end{tabular}


Supplementary data 2: Eleven predefined constructs used as an extraction tool and relating to the reporting effectiveness of reviews

\section{Aim of Review}

2 Theoretical basis (any stated theory)

3 Behaviour change technique (goal setting, problem solving)

4 location(s) or setting where the intervention occurred (local leisure centre, green spaces)

$5 \quad$ Intervention provider (should always be $3^{\text {rd }}$ party exercise provider as part of ERS)

6 Frequency of intervention (period of time including the number of sessions, their schedule, and their duration, intensity (if mentioned in initial studies or current review))

$7 \quad$ Mode of delivery (group, individual, supervised)

8 Characteristics of the participants (sex, disease or risk state)

9 Amount of studies within the review

10 Number of participants within the review / Sample sizes

11 Cost effectiveness if mentioned 
Supplementary data 3: Key characteristics of included systematic reviews

\begin{tabular}{|c|c|c|c|c|c|c|c|c|c|c|c|}
\hline \multicolumn{12}{|c|}{ Review lead author and year } \\
\hline $\begin{array}{c}\text { Characteristics } \\
\text { of reviews }\end{array}$ & $\begin{array}{l}\text { Arsenijevic } \\
\text { et al., } \\
2017\end{array}$ & $\begin{array}{l}\text { Campbell } \\
\text { et al., } \\
2015\end{array}$ & $\begin{array}{l}\text { Gidlow } \\
\text { et al., } \\
2005\end{array}$ & $\begin{array}{l}\text { Morgan } \\
\text { et al., } \\
2016\end{array}$ & $\begin{array}{l}\text { Morgan, } \\
2005\end{array}$ & $\begin{array}{l}\text { Orrow } \\
\text { et al., } \\
2012\end{array}$ & $\begin{array}{l}\text { Pavey } \\
\text { et al., } \\
2011 a\end{array}$ & $\begin{array}{l}\text { Pavey } \\
\text { et al., } \\
2012\end{array}$ & $\begin{array}{l}\text { Pavey } \\
\text { et al., } \\
2011 b\end{array}$ & $\begin{array}{l}\text { Sorensen } \\
\text { et al., } \\
2006\end{array}$ & $\begin{array}{l}\text { Williams } \\
\text { et al., } \\
2007\end{array}$ \\
\hline \multicolumn{12}{|c|}{ Type of review performed and its search period } \\
\hline Descriptive & $\checkmark$ & $\checkmark$ & & $\checkmark$ & $\checkmark$ & $\checkmark$ & $\checkmark$ & $\checkmark$ & $\checkmark$ & $\checkmark$ & $\checkmark$ \\
\hline Meta-analysis & $\checkmark$ & $\checkmark$ & & & & $\checkmark$ & $\checkmark$ & $\checkmark$ & $\checkmark$ & & $\checkmark$ \\
\hline $\begin{array}{l}\text { Economic } \\
\text { Evaluation }\end{array}$ & & $\checkmark$ & & & & & $\checkmark$ & & & & \\
\hline $\begin{array}{l}\text { Date period } \\
\text { searched }\end{array}$ & $\begin{array}{c}2000 \\
\text { onwards }\end{array}$ & $\begin{array}{c}\text { October } \\
2009- \\
\text { June } 2013\end{array}$ & $\begin{array}{l}\text { Origin - } \\
2003\end{array}$ & $\begin{array}{l}1995- \\
\text { June } \\
2015\end{array}$ & $\begin{array}{c}1966- \\
2002\end{array}$ & $\begin{array}{l}\text { Origin - } \\
\text { May } 2009\end{array}$ & $\begin{array}{c}1990- \\
\text { October } \\
2009\end{array}$ & $\begin{array}{l}\text { Origin - } \\
\text { October } \\
2009\end{array}$ & $\begin{array}{l}\text { Origin - } \\
\text { October } \\
2009\end{array}$ & $\begin{array}{c}1980- \\
\text { June } 2005\end{array}$ & $\begin{array}{l}\text { Origin - } \\
\text { March } \\
2007\end{array}$ \\
\hline \multicolumn{12}{|c|}{ Objectives and outcomes markers of review } \\
\hline $\begin{array}{c}\text { Overall } \\
\text { effectiveness } \\
\text { (increase of PA } \\
\text { levels) }\end{array}$ & $\checkmark$ & & & $\checkmark$ & $\checkmark$ & $\checkmark$ & & & $\checkmark$ & $\checkmark$ & $\checkmark$ \\
\hline $\begin{array}{c}\text { Clinical } \\
\text { effectiveness }\end{array}$ & & $\checkmark$ & & & & & $\checkmark$ & & $\checkmark$ & & \\
\hline $\begin{array}{c}\text { Cost } \\
\text { effectiveness }\end{array}$ & & $\checkmark$ & & & & & $\checkmark$ & & & $\checkmark$ & \\
\hline Uptake to ERS & & & & & & & $\checkmark$ & $\checkmark$ & & & \\
\hline $\begin{array}{c}\text { Attendance or } \\
\text { completion of } \\
\text { ERS }\end{array}$ & & & $\checkmark$ & $\checkmark$ & & & $\checkmark$ & $\checkmark$ & & & $\checkmark$ \\
\hline $\begin{array}{l}\text { Implementation } \\
\text { / design ERS }\end{array}$ & $\checkmark$ & & & & & & & & & $\checkmark$ & \\
\hline \multicolumn{12}{|c|}{ Reported inclusion criteria of reviews } \\
\hline
\end{tabular}




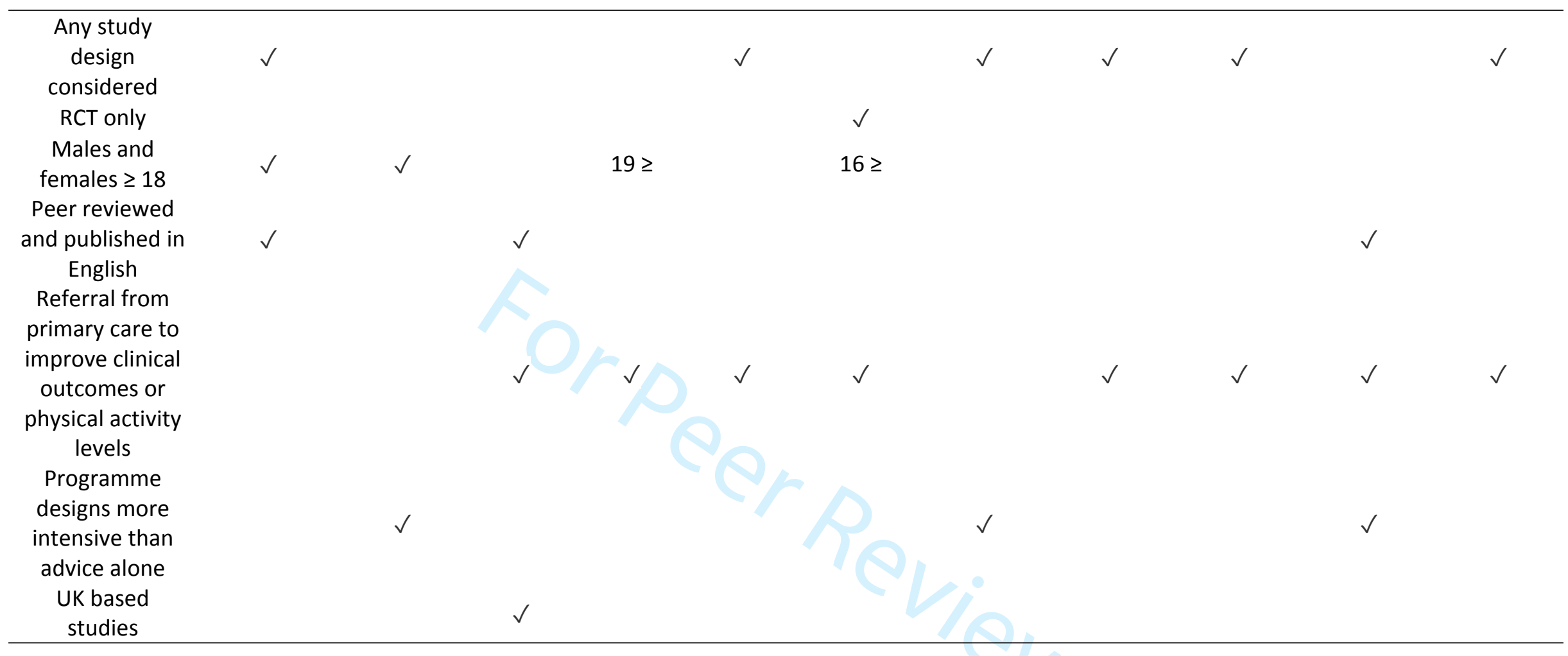


Supplementary data 4: Protocol to undertake systematic review of reviews.

Insufficient Reporting of Factors Associated with Exercise Referral Scheme Uptake, Attendance and Adherence: A Systematic Review of Reviews (Protocol)

\section{Review Question}

1) Describe definitions, rates, and participant characteristics associated with ERS uptake; 2) to describe definitions, rates, and participant characteristics associated with attendance at and adherence to ERS schemes; and 3) to describe theoretically informed approaches associated with ERS uptake and attendance.

\section{Searches}

We will systematically search the Databases:

- CINHAL, Medline, Embase, Cochrane Library, SportsDiscus, PsychInfo, SCOPUS, HMIC, AMED, Public Health Database, ASSIA, CRD databases, Prospero, Web of Knowledge, Campbell Collaboration Library and PubMed.

We will perform additional non-systematic searches of UpTodate, BMJ Best Practice and Dynamed. We will hand search reference lists of included articles for additional material. The searches will be restricted to English language, reviews published within peer viewed journals, and with no date restrictions.

\section{Type of Study to be included}

We will include systematic reviews that themselves have reviewed RCTs, observational studies, casecontrolled or other experimental studies and meta-analysis.

\section{Condition or domain being studied}

Exercise referral schemes. Commonly used terms within the literature are 'Exercise on Prescription' and 'GP Referral', which we will also be search for to ensure no data is missed. 


\section{Participants/population}

Inclusion: We will search for adults (18 years and over) of any gender who had been diagnosed or considered at risk of non-communicable disease (e.g. type 2 diabetes, hypertension, cardiovascular disease) by their primary care practitioner. Interventions that use participants that were sedentary with no further risk factors of non-communicable disease, but are used as a control groups will be included if reported separately.

Exclusion: We will exclude youths and children.

Intervention(s), exposure(s)

Inclusion: We will include systematic reviews that have reviewed interventions known as 'exercise referral', 'GP referral', or 'exercise on prescription'. Interventions promoting changes in physical activity behaviour via a direct referral from a health practitioner within primary care to a recognised exercise programme.

Exclusion: We will exclude reviews that report on interventions initiated from secondary care or are health-screening programmes.

\section{Primary outcomes}

- Define rates and participant characteristics associated with ERS uptake.

- Define rates and participant characteristics associated with attendance at, and, adherence to, ERS schemes.

- Report theoretically informed approaches associated with ERS uptake and attendance.

\section{Secondary outcomes}

- Establish what key features of ERS reporting go relatively under-reported.

\section{Data extraction (selection and coding)}

The PRISMA (Preferred Reporting Items for Systematic Reviews and Meta-Analyses) statement will guide the conduct of the narrative of the review of reviews. Results of the search strategy will be 
screened for duplicates by one researcher, with duplicates being removed. One author will independently examine titles and abstracts against the inclusion and exclusion criteria. Three authors will independently assess a randomised sub sample of papers, amounting to $20 \%$ of the total title and abstract sample. A group consensus will decide which papers will be progressed further to full text review and which to exclude. Full text review articles will be obtained and assessed by two authors against the inclusion and exclusion criteria. We will provide a detailed recording of the selection process, which we will report via a PRISMA flow diagram and 'Characteristics of excluded studies' table.

\section{Data to be extracted:}

- Aim of Review / study objectives

- Theoretical basis (any stated theory)

- Behaviour change technique (goal setting, problem solving)

- location(s) or setting where the intervention occurred (local leisure centre, green spaces)

- Intervention provider (should always be 3rd party exercise provider as part of ERS)

- Frequency of intervention (period of time including the number of sessions, their schedule and their duration, intensity (if mentioned in initial studies or current review))

- Mode of delivery (group, individual, supervised)

- Characteristics of the participants (sex, disease or risk state)

- Amount of studies within the review

- Number of participants within the review / sample sizes

- Cost effectiveness if mentioned

- Author name and date

- Reporting of attendance/ adherence rates

- Reporting on non-attenders

- Attendance figures, completion rates

- $\quad$ Key findings from reviews

\section{Risk of bias (quality) assessment}

Two authors will grade each review independently and empirically and discuss any discrepancies.

We will use The AMSTAR (A Measurement Tool to Assess Systematic Reviews) criteria to grade the reviews. Amstar is based on 11 questions and scores as reviews as follows: low quality (0-3), medium (4-7) and high (8-11). Question within the AMSTAR are as follows: 
1. Was an 'a priori' design provided?

2. Was there duplicate study selection and data extraction?

3. Was a comprehensive literature search performed?

4. Was the status of publication (i.e. grey literature) used as an inclusion criterion?

5. Was a list of studies (included and excluded) provided?

6. Were the characteristics of the included studies provided?

7. Was the scientific quality of the included studies assessed and documented?

8. Was the scientific quality of the included studies used appropriately in formulating conclusions?

9. Were the methods used to combine the findings of studies appropriate?

10. Was the likelihood of publication bias assessed?

11. Was the conflict of interest included?

\section{Strategy for data synthesis}

A table of results will display the extracted information by study. We will present the findings via a descriptive narrative of the key variables associated with uptake attendance and adherence to exercise referral schemes. 